\title{
Incorporation and washout of orally administered $n-3$ fatty acid ethyl esters in different plasma lipid fractions
}

\author{
Sonja D. Zuijdgeest-van Leeuwen ${ }^{1}$, Pieter C. Dagnelie ${ }^{2} *$, Trinet Rietveld ${ }^{1}$, J.Willem O. van den Berg \\ and J. H. Paul Wilson ${ }^{1}$ \\ ${ }^{1}$ Institute of Internal Medicine II, Erasmus University of Rotterdam, PO Box 1738, 3000 DR Rotterdam, The Netherlands \\ ${ }^{2}$ Department of Epidemiology, Maastricht University, PO Box 616, 6200 MD Maastricht, The Netherlands
}

(Received 22 September 1998 - Revised 29 March 1999 - Accepted 4 May 1999)

\begin{abstract}
The aim of the present study was to quantify the incorporation of eicosapentaenoic acid (EPA) and docosahexaenoic acid (DHA) into plasma lipids after oral administration of $n-3$ fatty acid ethyl esters, since little is known about the rate and pattern of incorporation into plasma lipid fractions. In addition, we aimed to obtain preliminary information regarding EPA half-life, which is needed to establish an optimal dosing schedule. Five healthy volunteers ingested two $8.5 \mathrm{~g}$ doses of $n-3$ fatty acid ethyl esters daily for $7 \mathrm{~d}$, supplying $6.0 \mathrm{~g}$ EPA/d and $5 \cdot 3 \mathrm{~g} \mathrm{DHA} / \mathrm{d}$. The fatty acid compositions of plasma phospholipids (PL), cholesteryl esters (CE) and triacylglycerols (TAG) were determined during supplementation and during a washout period of $7 \mathrm{~d}$. Halflives of EPA and DHA were calculated. The proportion of EPA in PL showed a 15-fold increase after $7 \mathrm{~d}(P<0.001)$, while DHA showed a smaller increase $(P<0.01)$. In CE, EPA also increased $(P<0.05)$, while DHA did not increase at all. Remarkably, incorporation of DHA into TAG was even higher than that of EPA. Half-life of EPA in PL ranged from 1.63 to $2.31 \mathrm{~d}$ (mean 1.97 (SE 0.15) d), whereas mean half-life of EPA in CE was 3.27 (SE 0.56) d. In three subjects, washout of EPA and DHA from TAG seemed to follow a bi-exponential pattern, with a short half-life $(<1 \mathrm{~d})$ in the initial phase and a half-life of several days in the second phase. In conclusion, EPA ethyl esters are rapidly incorporated into plasma lipids, especially into PL. The relatively long half-life of EPA in plasma would permit a dosing schedule with intervals of $\geqslant 12 \mathrm{~h}$ in supplementation studies.
\end{abstract}

Plasma lipids: n-3 Fatty acids: Ethyl esters

Beneficial health effects of $n-3$ fatty acids in fish oil have been described for several diseases. $n-3$ Fatty acids, especially eicosapentaenoic acid (20:5n-3; EPA) and docosahexaenoic acid (22:6n-3; DHA), are known to reduce plasma triacylglycerol (TAG) concentrations and may be beneficial in reducing the risk of atherosclerosis and inflammatory diseases (Simopoulos, 1991; Drevon, 1992).

Since fish oil contains a mixture of TAG with various fatty acids, the concentration of $n-3$ fatty acids is relatively low. Higher concentrations of EPA and DHA can be achieved by using a supplement of unesterified fatty acids. Ingestion of free fatty acids (FFA), however, causes gastrointestinal complaints and may be toxic (Beckermann et al. 1990). In contrast to FFA, no side-effects are induced by $n-3$ fatty acid ethyl esters (EE), and $\mathrm{EE}$ would therefore be appropriate for supplementation of diets with $n-3$ fatty acids. However, the absorption of EPA and DHA as EE may be lower than that of EPA and DHA as TAG or FFA, since previous studies have reported that the plasma incorporation of EPA- and DHA-EE is lower than the incorporation of EPA- and DHA-TAG after a single dose (el Boustani et al. 1987; Lawson \& Hughes, 1988; Beckermann et al. 1990) as well as during prolonged supplementation of $n-3$ fatty acids as EE (Hansen et al. 1993). However, other studies did not find any differences in incorporation between supplements (Reis et al. 1990; Nordoy et al. 1991; Krokan et al. 1993). The diversity of results may be partly explained by the fact that some studies measured EPA and DHA only in plasma TAG and not in plasma phospholipids (PL) or cholesteryl esters (CE). Before using $n-3$ fatty acid $E E$ in a clinical setting, it is important to determine the actual level of incorporation when using $n-3$ fatty acid EE.

In addition, information about the kinetics of EPA and DHA is needed to devise proper dosing schedules. However, only limited information is available about the rate of incorporation and the half-life of EPA and DHA in blood

\footnotetext{
Abbreviations: CE, cholesteryl esters; DHA, docosahexaenoic acid; EE, ethyl esters; EPA, eicosapentaenoic acid; MSC, model selection criterion; PL, phospholipids; TAG, triacylglycerols.

*Corresponding author: Dr Pieter Dagnelie, fax +31 43361 8685, email Dagnelie@epid.unimaas.nl
} 
and the information available is mainly restricted to single dose supplementation. A few studies investigating the rate of incorporation of EPA after a single dose of oral EPA-EE have reported maximal incorporation of EPA into TAG after 4-6h (el Boustani et al. 1987; Lawson \& Hughes, 1988; Nordoy et al. 1991). In studies with prolonged supplementation of $n-3$ fatty acid EE or TAG, EPA was found to reach its maximum level within 1 week (Hansen et al. 1993; Hodge et al. 1993). In contrast, the rise in DHA continued for several weeks (Hansen et al. 1993; Hodge et al. 1993). Although this difference in incorporation between EPA and DHA may be due to a difference in EPA or DHA concentration in the supplement, it may also be caused by a difference in metabolism between EPA and DHA. Some studies have shown that the decrease in EPA after cessation of supplementation is much more rapid than that of DHA (Hodge et al. 1993), but no information is available about the half-lives of EPA and DHA in plasma lipids.

The first aim of the present study was to determine the levels of incorporation of EPA and DHA in different plasma lipid fractions during supplementation with $n-3$ fatty acid EE. In addition, we attempted to quantify the half-lives of EPA and DHA in the different plasma lipid fractions.

\section{Subjects and methods}

\section{Subjects}

Study subjects were five healthy volunteers, four females and one male, of normal weight (BMI $20-25 \mathrm{~kg} / \mathrm{m}^{2}$ ) and aged between 23 and 30 years. None had a history of gastrointestinal complaints or metabolic or other serious diseases. All subjects consumed a normal Dutch diet, containing not more than one meal of fish per week. From 1 week before start of the protocol until the end of the study, subjects did not ingest any fish or fish products apart from the supplement. This study was approved by the Medical Ethical Committee of the University Hospital Dijkzigt, Rotterdam.

\section{Experimental protocol}

Duration of the study was $14 \mathrm{~d}: 7 \mathrm{~d}$ of $n-3$ fatty acid EE administration and $7 \mathrm{~d}$ of washout. On day 0 , subjects attended the outpatient department after an overnight fast. After $15 \mathrm{~min}$ rest, venous blood samples were taken into tubes containing EDTA. Thereafter, subjects consumed 8.5 g $n$-3 fatty acid EE as a fluid (Incromega E2573; Croda Oleochemicals, North Humberside, UK) twice daily with breakfast and supper respectively, for a period of $7 \mathrm{~d}$. To improve the taste, the $n-3$ fatty acid EE were mixed with two drops of peppermint oil just before consumption. The supplement contained 35.0 g EPA and $31.2 \mathrm{~g}$ DHA/ $100 \mathrm{~g}$ total fatty acids according to information from the supplier, resulting in intakes of $6 \mathrm{~g}$ EPA and $5.3 \mathrm{~g} \mathrm{DHA} / \mathrm{d}$. The fatty acid composition of the supplement is shown in Table 1 . Subsequently, fasting blood samples were taken during supplementation in the morning of days 1, 2, 3, 4 and 7 . To determine the rates of washout of EPA and DHA, blood sampling was continued on days 8, 9, 10, 11 and 14. Blood samples were placed on ice immediately after sampling and
Table 1. Contents ( $\mathrm{g} / 100 \mathrm{~g}$ total fatty acids) of the relevant fatty acids in the $n-3$ fatty acid ethyl ester supplement

\begin{tabular}{lr}
\hline Fatty acids & \\
\hline $18: 1 n-9$ & $4 \cdot 7$ \\
$18: 2 n-6$ & $1 \cdot 5$ \\
$18: 3 n-3$ & $0 \cdot 4$ \\
$20: 3 n-6$ & $0 \cdot 3$ \\
$20: 4 n-6$ & $2 \cdot 2$ \\
$20: 4 n-3$ & $1 \cdot 0$ \\
$20: 5 n-3$ (EPA) & $35 \cdot 0$ \\
$22: 5 n-3$ & $7 \cdot 5$ \\
$22: 6 n-3$ (DHA) & $31 \cdot 2$ \\
Other fatty acids & $19 \cdot 1$
\end{tabular}

EPA, eicosapentaenoic acid; DHA, docosahexaenoic acid.

centrifuged within $5 \mathrm{~min}$. Plasma was stored at $-70^{\circ}$ under $\mathrm{N}_{2}$ until analysis within 6 weeks.

\section{Fatty acid composition}

Plasma lipids were extracted using chloroform-methanol (2:1, v/v; Merck, Darmstadt, Germany) according to the method of Folch et al. (1956) in the presence of butylated hydroxytoluene $(1 \mathrm{mg} / \mathrm{ml})$ as antioxidant. Before extraction, internal standards were added in order to calculate absolute plasma concentrations of PL, CE and TAG (purity 96, 74 and $95 \%$ respectively). Plasma lipids were separated into PL, TAG and CE by TLC (silica plates; Merck, 5721) using hexane-diisopropylether-acetic acid $(60: 40: 3$, by vol.; Merck) as a developer. Fatty acids were converted into their methyl esters using a mixture of acetyl chloride (Merck) and methanol (Lillington et al. 1981). Fatty acid analyses were performed by GLC (Carlo Erba HRGC 5160) using a flame ionization detector, a Chrompack CP-sil 88 column (length $50 \mathrm{~m}$, diameter $0.25 \mathrm{~mm}$ ), an uncoated methyl deactivated retention gap, $\mathrm{He}$ as a carrier gas and 'on column' injection. Concentrations of individual fatty acids are presented as $\mathrm{mol} / 100 \mathrm{~mol}$ total fatty acids and not as absolute values, because of the statistically significant decreases in total plasma PL, CE and TAG concentrations.

\section{Total plasma triacylglycerols}

Plasma TAG concentrations were measured on days 0, 2, 7 and 11 using a standard enzymic assay from Boehringer Mannheim (no. 917; Mannheim, Germany).

\section{Total plasma free fatty acids}

Plasma FFA concentrations were measured on days 0, 2, 7 and 11 using an enzymic colorimetric method (NEFA C test kit; Wako Chemicals, Neuss, Germany).

\section{Total plasma phospholipids and cholesteryl esters}

Total plasma PL and CE were determined on all days by adding up the GLC peak areas of all fatty acids for the fraction concerned. 
Calculation of half-lives of eicosapentaenoic and docosahexaenoic acids

The Scientist ${ }^{\circledR}$ software package (MicroMath Scientific Software, UT, USA) was used for least squares fitting of the data during the washout period, using a monoexponential model and a bi-exponential model (Powell, 1970):

mono-exponential model:

concentration $=\mathrm{a} \times \exp ((-\mathrm{b}) \times$ time $)$,

half-life $=0 \cdot 693 / \mathrm{b}$;

bi-exponential model:

concentration $=\mathrm{a} 1 \times \exp ((-\mathrm{b} 1) \times$ time $)$ $+\mathrm{a} 2 \times \exp ((-\mathrm{b} 2) \times$ time $)$,

half-life $1=0 \cdot 693 / \mathrm{b} 1$,

half-life $2=0 \cdot 693 / \mathrm{b} 2$.

The model selection criterion (MSC) was defined by the formula:

$$
\text { MSC }=\ln \left(\frac{\sum_{i=1}^{n} w_{i}\left(Y_{o b s}-Y o b s\right)^{2}}{\sum_{i=1}^{n} w_{i}\left(Y_{o b s}-Y_{i} c_{i}\right)^{2}}\right)-\frac{2 p}{n},
$$

where $\mathrm{w}_{\mathrm{i}}$ are the weights applied to the points, $\mathrm{Yobs}_{\mathrm{i}}$ is the observed value of $\mathrm{Y}, \mathrm{Ycal}_{\mathrm{i}}$ is the calculated value of $\mathrm{Y}$ and $\mathrm{n}$ is the number of data points.

The MSC attempts to represent the 'information content' of a dataset, and indicates whether the model is suitable for the data or not. To calculate EPA and DHA half-lives, the model with the largest MSC is in principle the best model for the given data, and was used for data analysis. An MSC $<0.5$ was considered to be inadequate to give a good fit of the data. When one of the calculated parameter values was identical to the upper or lower limits purposely set as model constraints, data were considered invalid and were excluded from statistical analyses.

\section{Statistical analysis}

Based on an estimated mean change in EPA concentration during supplementation of $3 \mathrm{~mol} / 100 \mathrm{~mol}$, and a standard deviation of $1.4 \mathrm{~mol} / 100 \mathrm{~mol}$, the power of the study to detect a change of EPA was 0.94 using Student's paired $t$ test. Results are expressed as means with their standard errors. Changes in plasma lipid concentrations were tested by Student's paired $t$ test. For the figures, data were analysed by repeated measures analysis of covariance using the general linear mixed model (Proc Mixed procedure) of the Statistical Analysis Systems statistical software package (SAS Institute Inc., Cary, NC, USA). Compound symmetry was assumed and time was the only independent variable in the model. $P$ values less than 0.05 were considered to be significant.

\section{Results}

n-3 Fatty acid EE were well tolerated by all subjects. However, when belching occurred, a fishy smell or taste was noted. All subjects completed the study, but in one subject the blood sample of day 4 could not be taken. During supplementation, concentrations of individual plasma lipid fractions showed moderate to substantial changes (Table 2). Plasma TAG concentrations decreased significantly during supplementation (days 2 and $7 ; P<0 \cdot 05$ ), returning to near baseline values after $4 \mathrm{~d}$ of washout (day 11). Plasma FFA concentrations also decreased significantly within $2 \mathrm{~d}$ of supplementation $(P=0.01)$ and remained low during supplementation, although the difference from baseline after $7 \mathrm{~d}$ failed to reach statistical significance $(P=0 \cdot 07)$. Both plasma PL and CE concentrations showed significant decreases during $n$-3 fatty acid EE supplementation.

Consumption of $n-3$ fatty acid EE for 1 week resulted in considerable changes in plasma fatty acid composition. In plasma PL, a highly significant increase of EPA (20:5n-3) was observed, approaching a plateau level after approximately $4 \mathrm{~d}$ (Fig. 1). During washout, the EPA concentration decreased rapidly, with an almost complete return to

Table 2. Plasma concentrations ( $\mathrm{mmol} / \mathrm{l})$ of total phospholipids (PL), cholesteryl esters (CE), triacylglycerols (TAG) and free fatty acids (FFA) before (day 0), during (days 1-7) and after (days 8-14) consumption of fish oil ethyl esters by healthy volunteers

(Mean values with their standard errors for five subjects)

\begin{tabular}{|c|c|c|c|c|c|c|c|c|}
\hline \multirow[b]{2}{*}{ Day } & \multicolumn{2}{|c|}{ PL†‡ } & \multicolumn{2}{|c|}{ CE† } & \multicolumn{2}{|c|}{ TAG } & \multicolumn{2}{|c|}{ FFA } \\
\hline & Mean & SE & Mean & $\overline{\mathrm{SE}}$ & Mean & SE & Mean & SE \\
\hline 0 & 1.26 & 0.09 & $2 \cdot 29$ & 0.21 & 0.84 & 0.15 & 0.29 & 0.04 \\
\hline 1 & 1.21 & 0.09 & 2.01 & 0.13 & & & & \\
\hline 2 & $1 \cdot 16$ & 0.08 & 1.85 & 0.13 & $0.50^{*}$ & 0.13 & $0.12^{*}$ & 0.02 \\
\hline 3 & 1.10 & 0.09 & 1.86 & 0.16 & & & & \\
\hline 4 & 1.11 & 0.11 & 1.75 & 0.14 & & & & \\
\hline 7 & 1.15 & 0.08 & 1.85 & 0.11 & $0.51^{*}$ & 0.04 & 0.14 & 0.05 \\
\hline 8 & 1.19 & 0.08 & 1.89 & 0.10 & & & & \\
\hline 9 & 1.26 & 0.08 & 1.95 & 0.08 & & & & \\
\hline 10 & 1.25 & 0.10 & 1.92 & 0.09 & & & & \\
\hline 11 & 1.31 & 0.09 & 1.98 & 0.07 & 0.72 & 0.09 & 0.27 & 0.03 \\
\hline 14 & 1.29 & 0.07 & 2.04 & 0.06 & & & & \\
\hline
\end{tabular}

Mean values were significantly different from those for day $0:{ }^{*} P<0.05$ (paired $t$ test).

† Significant decrease during supplementation $(P<0.05$; repeated measures).

$\ddagger$ Significant increase during washout period $(P<0.05$; repeated measures). 


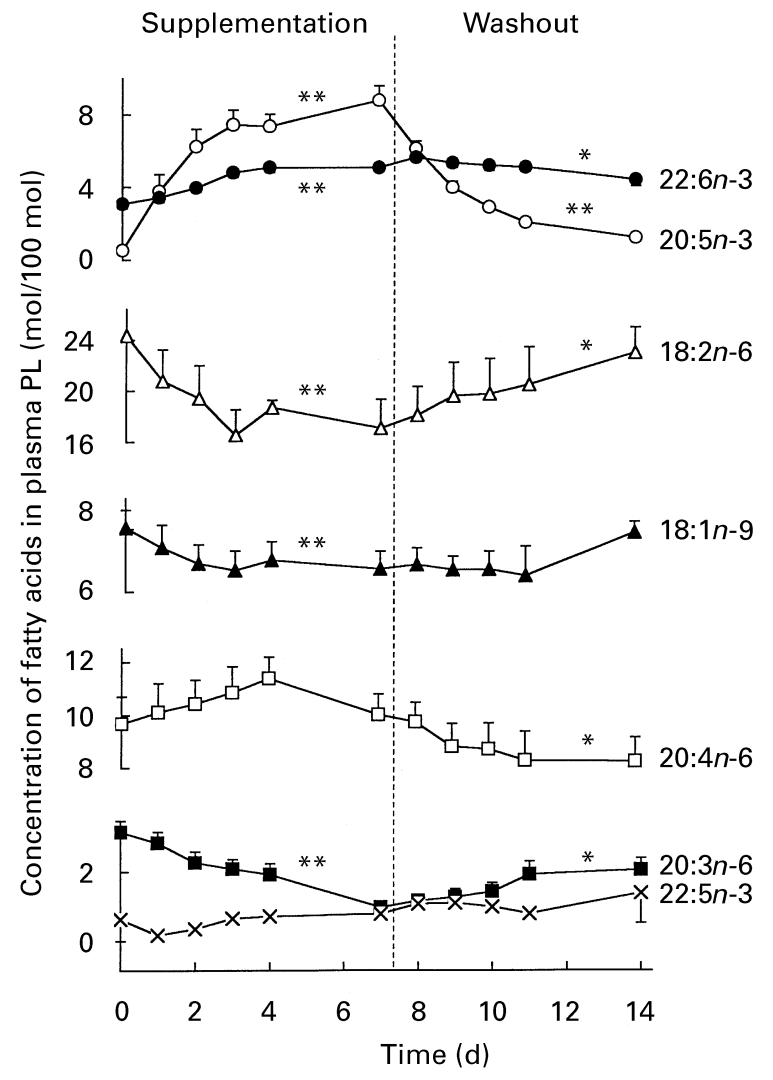

Fig. 1. The concentration of different fatty acids in plasma phospholipids $(\mathrm{PL})(\mathrm{mol} / 100 \mathrm{ml}$ total fatty acids in the $\mathrm{PL}$ fraction) during daily intake of $n-3$ fatty acid ethyl esters $(17 \mathrm{~g} / \mathrm{d})$ and during the washout period. Values are means for five subjects, with their standard errors represented by vertical bars. Significant trend by repeated measures analysis: ${ }^{*} P<0.05,{ }^{* *} P<0.0001$.

baseline values after $7 \mathrm{~d}$ of washout. A significant increase was also found for DHA $(22: 6 n-3)$ concentrations in plasma PL, although this increase was much less pronounced than that of EPA. Arachidonic acid (20:4n-6) also showed a slight increase, which reached significance only on days 2 and 3 when using the paired $t$ test. In contrast, levels of $18: 2 n-6,18: 1 n-9$ and $20: 3 n-6$ in plasma PL decreased significantly during supplementation, and returned to initial levels by day 14 . No significant changes in plasma PL were found for $22: 5 n-3$.

In plasma $\mathrm{CE}$, changes in fatty acid composition were less pronounced than in plasma PL (Fig. 2). Incorporation of EPA into the CE fraction was less rapid than in the PL fraction, and the EPA concentration did not reach a plateau level within $7 \mathrm{~d}$. Subsequently, the decrease of EPA during washout was less rapid than in PL. DHA incorporation into CE was minimal, while the proportion of $20: 4 n-6$ increased significantly. A small and insignificant increase of $20: 3 n-6$ and $22: 5 n-3$ was found. No changes were detected in the levels of $18: 2 n-6$ and $18: 1 n-9$ in plasma CE during supplementation.

A completely different pattern of incorporation was observed for plasma TAG (Fig. 3): here, DHA was incorporated to an even higher extent than EPA, in contrast to the

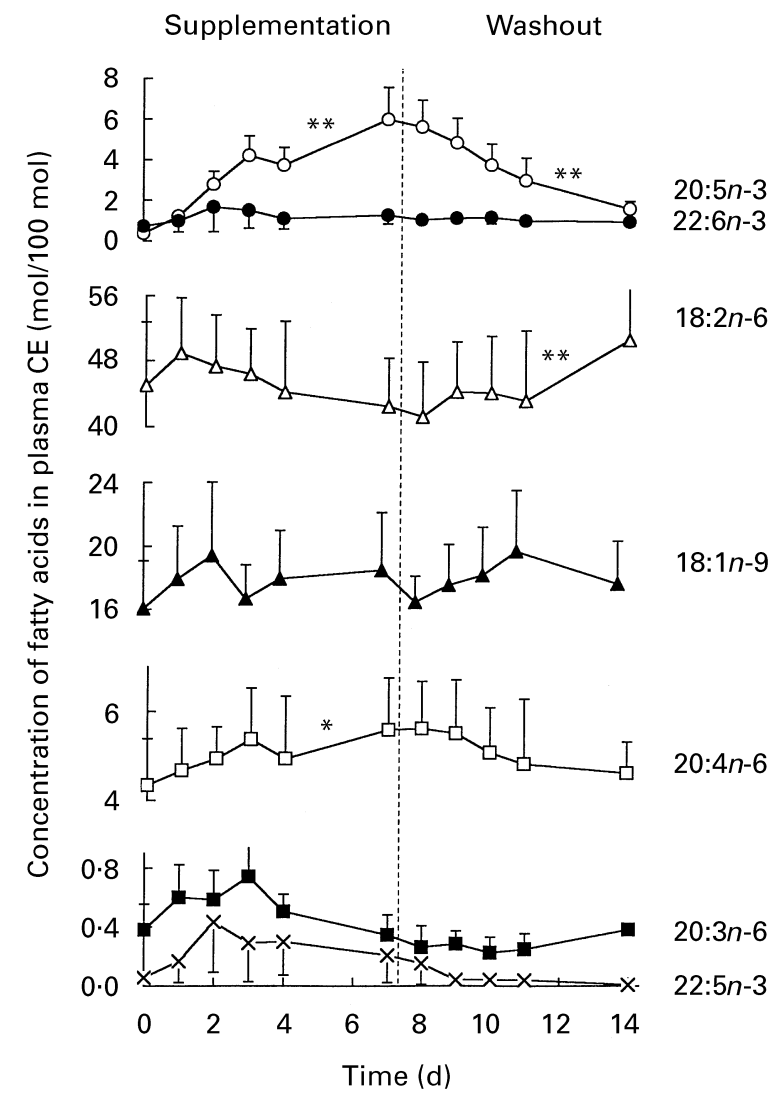

Fig. 2. The concentration of different fatty acids in plasma cholesteryl esters (CE) ( $\mathrm{mol} / 100 \mathrm{ml}$ total fatty acids in the CE fraction) during daily intake of $n-3$ fatty acid ethyl esters $(17 \mathrm{~g} / \mathrm{d})$ and during the washout period. Values are means for five subjects, with their standard errors represented by vertical bars. Significant trend by repeated measures analysis: ${ }^{*} P<0.05,{ }^{* *} P<0.0001$.

incorporation into PL and CE. Both fatty acids showed a rapid decrease during washout. EPA had completely returned to baseline on day 14, while DHA was still significantly increased by this time. While incorporation of $22: 5 n-3$ into PL and CE was minimal, a fivefold increase of $22: 5 n-3$ was detected in TAG, reaching a plateau value within $2 \mathrm{~d}$. This was followed by a rapid return to baseline values during washout. In addition, $20: 4 n-6$ showed a significant increase on days 2 and 3 (paired $t$ test), while $20: 3 n-6$ was significantly increased at day 1 . This was accompanied by a decrease in $18: 1 n-9$, which only reached significance on day 3 (paired $t$ test). Again, no significant changes in the levels of $18: 2 n-6$ in TAG were found during the study.

Plasma half-life of EPA could be calculated in all plasma lipid fractions, but calculation of half-life of DHA was only possible in plasma TAG, due to the high scatter of data in plasma PL and the small changes of DHA in plasma CE. Individual washout curves are shown in Fig. 4.

The washout of EPA from plasma PL was well fitted by a mono-exponential model, resulting in an EPA half-life ranging from 1.63 to $2.31 \mathrm{~d}$ (mean 1.97 (SE 0.15) d) (Table 3). The high MSC value showed that the model 


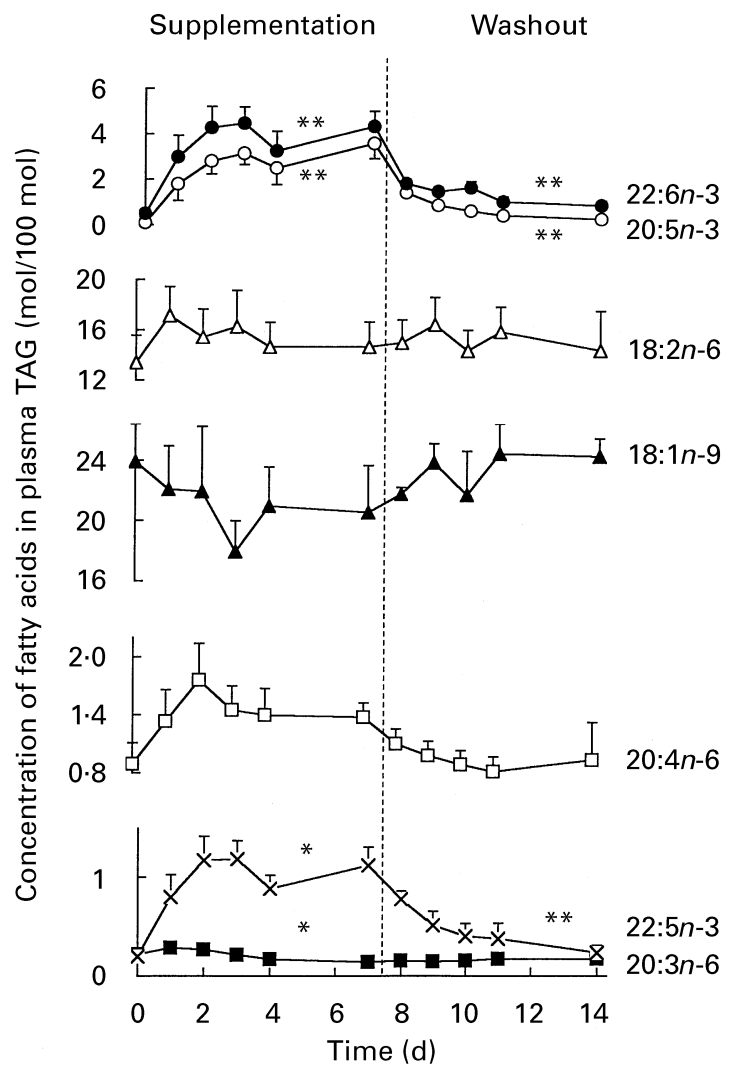

Fig. 3. The concentration of different fatty acids in plasma triacylglycerols (TAG) (mol/100 mol total fatty acids in the TAG fraction) during daily intake of $n-3$ fatty acid ethyl esters $(17 \mathrm{~g} / \mathrm{d})$ and during the washout period. Values are means for five subjects, with their standard errors represented by vertical bars. Significant trend by repeated measures analysis: ${ }^{*} P<0.05,{ }^{* *} P<0.0001$.

used was suitable for the data. In plasma CE, data were also best fitted mono-exponentially, although the MSC value was lower than in PL due to the higher intra-individual scatter. Half-life of EPA in CE could be calculated in four subjects and ranged from 1.63 to $4.08 \mathrm{~d}$ (mean 3.27 (SE $0.56) \mathrm{d}$ ).

The decrease of EPA in plasma TAG was initially fitted by the mono-exponential model (Table 3). Half-life of EPA in TAG could be calculated in four subjects and ranged from 0.47 to $1.63 \mathrm{~d}$ (mean $1.12 \mathrm{~d}$ ). However, in three of them, the MSC value showed an increase when fitting the data with a bi-exponential model, whereas in the fourth subject, the use of a bi-exponential model was not possible. In the three subjects, the initial half-life of EPA ranged from 0.11 to $0.77 \mathrm{~d}$ (mean 0.41 (SE 0.19) d), whereas half-life in the second phase was $1.29-4 \cdot 17 \mathrm{~d}$ (mean 2.92 (SE 0.86) d).

For the decrease of DHA in plasma TAG, comparable results were found (Table 3 ). A half-life ranging from 0.89 to $2.57 \mathrm{~d}$ was found with a mean of $1.63 \mathrm{~d}$ when using the mono-exponential model. Use of the bi-exponential model resulted in an increase of the MSC value. The initial half-life of DHA in plasma TAG ranged from 0.18 to $0.38 \mathrm{~d}$, followed by a half-life of $3 \cdot 81-5 \cdot 32 \mathrm{~d}$ in the second phase (mean 4.75 (SE 0.33)d).

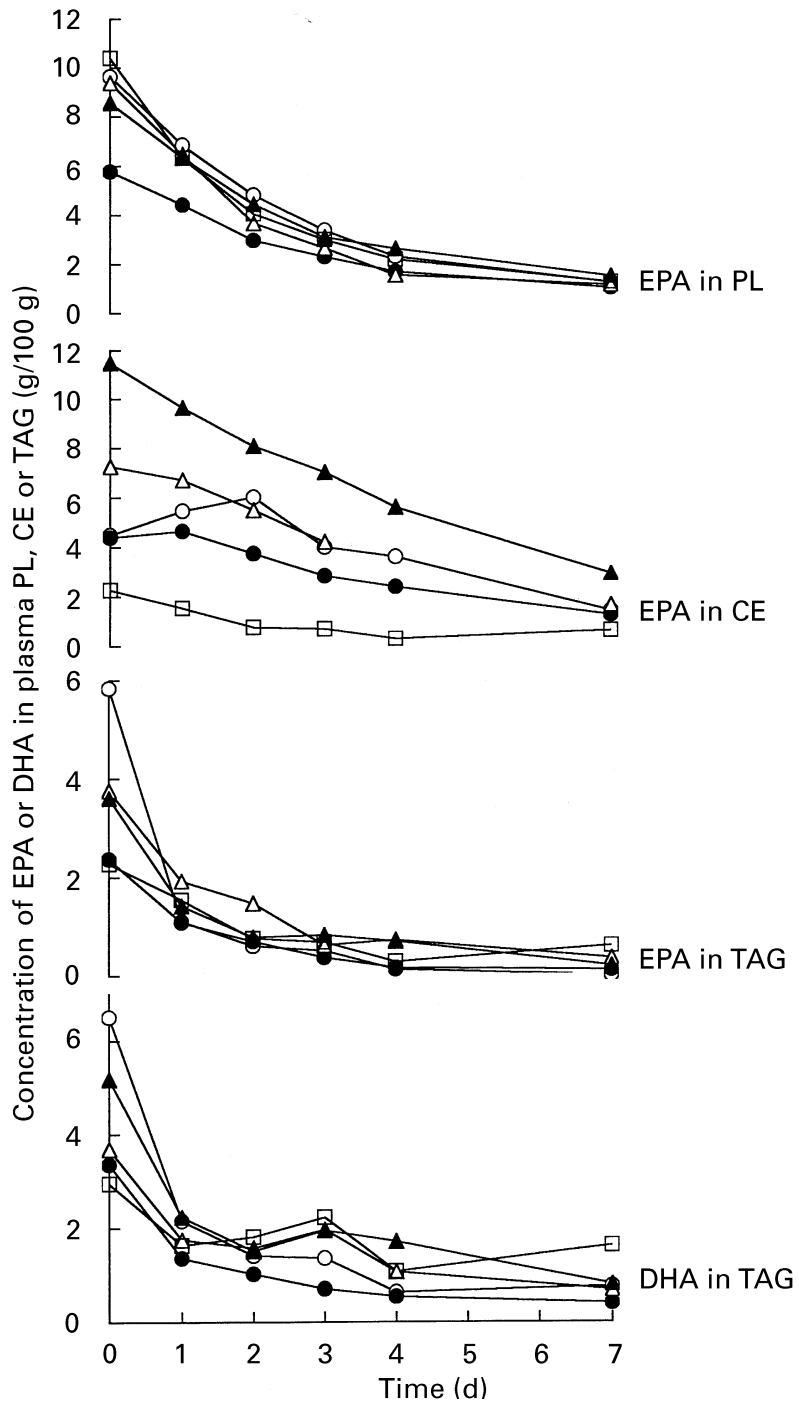

Fig. 4. Individual washout curves of eicosapentaenoic acid (EPA) from plasma phospholipids (PL), cholesteryl esters (CE) and triacylglycerols (TAG), and of docosahexaenoic acid (DHA) from plasma TAG in subjects who had consumed a supplement of $n-3$ fatty acid ethyl esters $(17 \mathrm{~g} / \mathrm{d})$ for $7 \mathrm{~d}$.

\section{Discussion}

Our study demonstrates that $n-3$ fatty acid EE are absorbed from the intestine, as was shown by the significant increases of EPA and DHA in different plasma lipid fractions during supplementation. In general, a relatively consistent pattern of incorporation and washout was shown in most lipid fractions with small deviations between subjects.

EPA was incorporated into PL and CE, as well as into TAG. In contrast, the DHA incorporation pattern was quite different: a very low incorporation into $\mathrm{CE}$, but a high incorporation into TAG which was even higher than that of EPA. Although not all data could be used for the calculation of plasma half-lives, this study provides preliminary information regarding washout of EPA and DHA from plasma. During washout, the decrease of EPA in PL and CE was found to be mono-exponential with mean half-lives of 1.97 and $3.27 \mathrm{~d}$ respectively. In plasma TAG, however, the data for EPA and DHA were better fitted by a bi-exponential 
Table 3. Half-lives of eicosapentaenoic acid (EPA) and docosahexaenoic acid (DHA) in different plasma lipid fractions, calculated by mono-exponential and bi-exponential models

\begin{tabular}{|c|c|c|c|c|c|c|}
\hline & \multirow[b]{2}{*}{ Subject } & \multicolumn{2}{|c|}{ Mono-exponential } & \multicolumn{3}{|c|}{ Bi-exponential } \\
\hline & & Half-life & $\mathrm{MSC}^{*}$ & Half-life 1 & Half-life 2 & $\mathrm{MSC}^{*}$ \\
\hline EPA in PL & $\begin{array}{l}1 \\
2 \\
3 \\
4 \\
5 \\
\text { Mean } \\
\text { SE }\end{array}$ & $\begin{array}{l}2.02 \\
2.31 \\
1.63 \\
2.27 \\
1.65 \\
1.97 \\
0.16\end{array}$ & $\begin{array}{l}5.1 \\
3.9 \\
3.6 \\
3.8 \\
3.5\end{array}$ & $\begin{array}{l}-\dagger \\
- \\
- \\
- \\
-\end{array}$ & $\begin{array}{l}- \\
- \\
- \\
- \\
-\end{array}$ & $\begin{array}{l}- \\
- \\
- \\
- \\
-\end{array}$ \\
\hline EPA in CE & $\begin{array}{l}1 \\
2 \\
3 \\
4 \\
5 \\
\text { Mean } \\
\text { SE }\end{array}$ & $\begin{array}{c}\ddagger \\
4.08 \\
3.59 \\
3.80 \\
1.63 \\
3.27 \\
0.56\end{array}$ & $\begin{array}{c}- \\
1.9 \\
2.9 \\
4.7 \\
1.5\end{array}$ & $\begin{array}{l}-\dagger \\
- \\
- \\
- \\
-\end{array}$ & $\begin{array}{l}- \\
- \\
- \\
- \\
-\end{array}$ & $\begin{array}{l}- \\
- \\
- \\
- \\
-\end{array}$ \\
\hline EPA in TAG & $\begin{array}{l}1 \\
2 \\
3 \\
4 \\
5 \\
\text { Mean } \\
\text { SE }\end{array}$ & $\begin{array}{l}0.47 \\
1.05 \\
1.37 \\
1.06 \\
1.63 \\
1.12 \\
0.19\end{array}$ & $\begin{array}{l}3.6 \\
4.0 \\
2.7 \\
2.0 \\
1.5\end{array}$ & $\begin{array}{c}0.11 \\
\S \\
0.77 \\
0.33 \\
\uparrow \\
0.41 \\
0.19\end{array}$ & $\begin{array}{c}1.29 \\
\S \\
4.17 \\
3.32 \\
\uparrow \\
2.92 \\
0.86\end{array}$ & $\begin{array}{c}5.4 \\
- \\
2.8 \\
3.6 \\
-\end{array}$ \\
\hline DHA in TAG & $\begin{array}{r}1 \\
2 \\
3 \\
4 \\
5 \\
\text { Mean } \\
\text { SE }\end{array}$ & $\begin{array}{c}0.89 \\
1.18 \\
2.57 \\
1.89 \\
\ddagger \\
1.63 \\
0.38\end{array}$ & $\begin{array}{c}1.9 \\
2.0 \\
0.9 \\
0.9 \\
-\end{array}$ & $\begin{array}{c}0.35 \\
0.38 \\
0.35 \\
0.18 \\
\neq \\
0.31 \\
0.04\end{array}$ & $\begin{array}{c}4.82 \\
3.81 \\
5.06 \\
5.32 \\
\neq \\
4.75 \\
0.33\end{array}$ & $\begin{array}{c}3 \cdot 5 \\
4 \cdot 4 \\
1 \cdot 1 \\
2 \cdot 2 \\
-\end{array}$ \\
\hline
\end{tabular}

PL, phospholipids; CE, cholesteryl esters; TAG, triacylglycerols; MSC, model selection criterion.

* MSC is an indicator of the suitability of the model; the higher the value, the better the model.

†No bi-exponential fit possible due to good fit of mono-exponential model.

$\ddagger$ No suitable fit due to high scatter of data.

$\S$ No suitable fit because parameter value was equal to estimated upper constraint.

ๆ No suitable fit due to negative value of half-life 2 .

washout model, with a short initial half-life of less than $1 \mathrm{~d}$, followed by a longer half-life of several days.

The incorporation of EPA was considerably higher than the incorporation of DHA into plasma lipids in the present study. This finding is consistent with results from other studies investigating the incorporation of $n-3$ fatty acid TAG (Nordoy et al. 1991; Hansen et al. 1993) as well as studies in which EE were used (Subbaiah et al. 1993; Gibney \& Daly, 1994; Leaf et al. 1995). Only Blonk et al. (1990), who used a supplement of $n-3$ fatty acid EE, did not find any difference in incorporation between EPA and DHA into plasma PL after 12 weeks of supplementation. In these studies, supplementation ranged from a single dose (Gibney \& Daly, 1994) to 1 year of supplementation (Leaf et al. 1995). The pattern of incorporation of different fatty acids appeared to be independent of the different fish oil types and dosages. Sadou et al. (1995) investigated whether the intramolecular structure of $n-3$ fatty acid TAG affects the incorporation of EPA and DHA. They found that DHA (predominantly situated at the $s n-2$ position) was mainly incorporated into plasma TAG, while EPA (predominantly situated at $s n-1 / 3$ ) was mainly incorporated into plasma PL (Sadou et al. 1995). They concluded that the difference in incorporation was due to the intramolecular structure. However, our study shows that a supplement containing EE instead of TAG resulted in a similar plasma distribution, which would suggest that the incorporation pattern is the result of properties of EPA and DHA rather than of the position of the fatty acid on the glycerol backbone.

A more plausible explanation for the difference in incorporation between EPA and DHA was given by Subbaiah et al. (1993). These authors suggested that the difference is caused by the fact that EPA and DHA are competitors for the enzymic transfer of fatty acids from PL to CE. The affinity of the enzyme lecithin-cholesterol acyltransferase is higher for EPA than for DHA. Thus, the transfer of EPA from PL to CE was higher compared with DHA. This would explain why, in our study, incorporation of EPA into CE was high, while the incorporation of DHA into CE was negligible. It would also explain why DHA incorporation into PL was more rapid than into $\mathrm{CE}$, a finding that has also been described in other studies (Subbaiah et al. 1993; Sadou et al. 1995; Brossard et al. 1996).

When the transfer of DHA from PL to CE is reduced, a relative accumulation of DHA in PL would be expected. Indeed, in our study DHA in PL was still increasing after 1 week of supplementation. However, the increase of DHA in PL was less rapid than the increase of EPA. This could be explained by a relatively high clearance of DHA compared with EPA from the plasma towards the adipose tissue, since DHA has been suggested to be the preferred storage form of n-3 fatty acids (Kasim-Karakas, 1995). 
$n$-3 Fatty acid supplementation was also shown to induce changes in fatty acids other than EPA and DHA in the present study. We observed a small increase in $20: 4 n-6$ in plasma PL on days 2 and 3, followed by a decrease in concentration. It is likely that the observed increase is the result of the small amount of $20: 4 n-6$ in the supplement. However, this increase does not correspond to that seen in other studies, which generally report a decrease in $20: 4 n-6$ during n-3 fatty acid supplementation (von Schacky et al. 1985; Reis et al. 1990; Hansen et al. 1993; Krokan et al. 1993; Marangoni et al. 1993; Prisco et al. 1996), most probably as a consequence of competition between the $n-3$ and $n-6$ fatty acids. Yet, when examining literature data in detail, some of these studies show a temporary increase in 20:4n-6 during the first week of supplementation (von Schacky et al. 1985; Reis et al. 1990; Hansen et al. 1993), which is in accordance with our findings. This observation, combined with the significant decrease of $18: 2 n-6$ in PL, indicates that incorporation of EPA and DHA into PL in the first days of supplementation mainly occurs at the expense of $18: 2 n-6$ and not of $20: 4 n-6$.

Not only was the incorporation level of EPA higher than that of DHA, the incorporation rate was also higher. Similarly, the decline during washout was more rapid for EPA than for DHA. The same finding has also been described by others (Marsen et al. 1992; Hodge et al. 1993; Marangoni et al. 1993; Subbaiah et al. 1993) who showed that DHA had not returned to baseline values within 4 weeks of washout. So far, no information has been available about the washout rates in the separate plasma lipid fractions. In our present study, the decrease of EPA in the PL and CE fractions was mono-exponential, implicating that EPA probably originates from one pool, i.e. plasma PL. Unfortunately, it was not possible to calculate half-life of DHA in PL and CE due to the high intraindividual scatter and the slow rate of washout.

As for plasma TAG, our results would indicate a biexponential washout of EPA as well as DHA. The slow decline in the second phase of washout may be due to the release of $n$ - 3 fatty acids from body stores. EPA and DHA are incorporated into TAG by the liver to be incorporated into VLDL and to re-enter the plasma pool. The half-life in the second phase is longer for DHA than for EPA, which is consistent with the notion that DHA is the main storage form of the $n-3$ fatty acids (Kasim-Karakas, 1995).

In summary, we conclude that $n-3$ fatty acid EE are sufficiently well incorporated into plasma lipids to allow their use in clinical practice. Our results suggest that the washout of EPA and DHA from plasma PL and CE is monoexponential, but that EPA and DHA washout from TAG may follow a bi-exponential pattern. Based on the relatively long half-life of the $n-3$ fatty acids in plasma, we conclude that supplementation with $6 \mathrm{~g}$ EPA in a frequency of one dose per $24 \mathrm{~h}$ would probably be sufficient to obtain a substantial increase in plasma EPA and DHA levels.

\section{Acknowledgements}

This work was supported by Numico Research BV, Wageningen, The Netherlands.

\section{References}

Beckermann B, Beneke M \& Seitz I (1990) Vergleich der Bioverfugbarkeit von Eicosapentaensaure und Docosahexaensaure aus Triglyceriden, freien Fettsauren und Ethylestern bei Probanden (Comparative bioavailability of eicosapentaenoic acid and docosahexaenoic acid from triacylglycerols, free fatty acids and ethyl esters in volunteers). Arzneimittelforschung 40, 700-704.

Blonk MC, Bilo HJ, Nauta JJ, Popp-Snijders C, Mulder C \& Donker AJ (1990) Dose-response effects of fish-oil supplementation in healthy volunteers. American Journal of Clinical Nutrition 52, 120-127.

Brossard N, Croset M, Pachiaudi C, Riou JP, Tayot JL \& Lagarde M (1996) Retroconversion and metabolism of $\left[{ }^{13} \mathrm{C}\right] 22: 6 n-3$ in humans and rats after intake of a single dose of $\left[{ }^{13} \mathrm{C}\right] 22: 6 n-3-$ triacylglycerols. American Journal of Clinical Nutrition 64, 577-586.

Drevon CA (1992) Marine oils and their effects. Nutrition Reviews 50, 38-45.

el Boustani S, Colette C, Monnier L, Descomps B, Crastes de Paulet A \& Mendy F (1987) Enteral absorption in man of eicosapentaenoic acid in different chemical forms. Lipids 22 , 711-714.

Folch J, Lees M \& Stanley GHS (1956) A simple method for the isolation and purification of total lipides from animal tissues. Journal of Biological Chemistry 226, 497-509.

Gibney MJ \& Daly E (1994) The incorporation of $n-3$ polyunsaturated fatty acids into plasma lipid and lipoprotein fractions in the postprandial phase in healthy volunteers. European Journal of Clinical Nutrition 48, 866-872.

Hansen JB, Olsen JO, Wilsgard L, Lyngmo V \& Svensson B (1993) Comparative effects of prolonged intake of highly purified fish oils as ethyl ester or triglyceride on lipids, haemostasis and platelet function in normolipaemic men. European Journal of Clinical Nutrition 47, 497-507.

Hodge J, Sanders K \& Sinclair AJ (1993) Differential utilization of eicosapentaenoic acid and docosahexaenoic acid in human plasma. Lipids 28, 525-531.

Kasim-Karakas SE (1995) Impact of $n$-3 fatty acids on lipoprotein metabolism. Current Opinion in Lipidology 6, 167-171.

Krokan HE, Bjerve KS \& Mork E (1993) The enteral bioavailability of eicosapentaenoic acid and docosahexaenoic acid is as good from ethyl esters as from glyceryl esters in spite of lower hydrolytic rates by pancreatic lipase in vitro. Biochimica et Biophysica Acta 1168, 59-67.

Lawson LD \& Hughes BG (1988) Human absorption of fish oil fatty acids as triacylglycerols, free acids, or ethyl esters. Biochemical and Biophysical Research Communications 152, 328-335.

Leaf DA, Connor WE, Barstad L \& Sexton G (1995) Incorporation of dietary $n-3$ fatty acids into the fatty acids of human adipose tissue and plasma lipid classes. American Journal of Clinical Nutrition 62, 68-73.

Lillington JM, Trafford DJ \& Makin HL (1981) A rapid and simple method for the esterification of fatty acids and steroid carboxylic acids prior to gas-liquid chromatography. Clinica Chimica Acta 111, 91-98.

Marangoni F, Angeli MT, Colli S, Eligini S, Tremoli E, Sirtori CR \& Galli C (1993) Changes of $n-3$ and $n-6$ fatty acids in plasma and circulating cells of normal subjects, after prolonged administration of 20:5 (EPA) and 22:6 (DHA) ethyl esters and prolonged washout. Biochimica et Biophysica Acta 1210, 5562.

Marsen TA, Pollok M, Oette K \& Baldamus CA (1992) Pharmacokinetics of omega-3-fatty acids during ingestion of fish oil preparations. Prostaglandins Leukotrienes and Essential Fatty Acids 46, 191-196. 
Nordoy A, Barstad L, Connor WE \& Hatcher L (1991) Absorption of the $n-3$ eicosapentaenoic and docosahexaenoic acids as ethyl esters and triglycerides by humans. American Journal of Clinical Nutrition 53, 1185-1190.

Powell MJD (1970) A FORTRAN subroutine for solving system of nonlinear algebraic equations. In Numerical Methods for Nonlinear Algebraic Equations, pp. 115-161 [P Robinowitz, editor]. New York, NY: Gordon \& Breach Science Publishers.

Prisco D, Filippini M, Francalanci I, Paniccia R, Gensini GF, Abbate K \& Neri Serneri GG (1996) Effect of $n$-3 polyunsaturated fatty acid intake on phospholipid fatty acid composition in plasma and erythrocytes. American Journal of Clinical Nutrition 63, 925-932.

Reis GJ, Silverman DI, Boucher TM, Sipperly ME, Horowitz GL, Sacks FM \& Pasternak RC (1990) Effects of two types of fish oil supplements on serum lipids and plasma phospholipid fatty acids in coronary artery disease. American Journal of Cardiology 66, 1171-1175.
Sadou H, Leger CL, Descomps B, Barjon JN, Monnier L \& Crastes de Paulet A (1995) Differential incorporation of fish-oil eicosapentaenoate and docosahexaenoate into lipids of lipoprotein fractions as related to their glyceryl esterification: a short-term (postprandial) and long-term study in health. American Journal of Clinical Nutrition 62, 1193-1200.

Simopoulos AP (1991) Omega-3 fatty acids in health and disease and in growth and development. American Journal of Clinical Nutrition 54, 438-463.

Subbaiah PV, Kaufman D \& Bagdade JD (1993) Incorporation of dietary $n-3$ fatty acids into molecular species of phosphatidyl choline and cholesteryl ester in normal human plasma. American Journal of Clinical Nutrition 58, 360-368.

von Schacky C, Fischer S \& Weber PC (1985) Long-term effects of dietary marine omega-3 fatty acids upon plasma and cellular lipids, platelet function, and eicosanoid formation in humans. Journal of Clinical Investigation 76, 1626-1631. 